\title{
Una aproximación a la dinámica local en Lima: factores a considerar en el análisis de su situación actual
}

\section{An approach to local dynamics in Lima: factors to consider in the analysis of the current situation}

\section{Jhasmin Camarena Miranda (*)}

Pontificia Universidad Católica del Perú

ORCID: 0000-0003-1536-0706

Fecha de recepción: 20 de mayo

Fecha de aceptación: 5 de agosto

ISSN en línea: $2415-2498$

Camarena Miranda, J. (2019) «Una aporximación a la dinámica loca en Lima: factores a considerar en el análisis de su situación actual». Politai: Revista de Ciencia Política, Año 10, primer semestre, № 19: pp.98-128.

DOI: https://doi.org/10.18800/politai.201901.004

*Licenciada en Ciencia Política y Gobierno de la Pontificia Universidad Católica del Perú. 


\section{Sumilla}

A partir de la exposición mediática de la cantidad de autoridades y exautoridades procesadas por casos de corrupción a nivel regional y local en el 2014 se retomó el interés sobre lo que ocurría en estos niveles de gobierno. Frente a ello, se tomó como medida la eliminación de la posibilidad de reelección inmediata en cargos de elección popular. Sin embargo, la comprensión de la problemática requiere un análisis de las dinámicas que existen en estos niveles para identificar los factores que la configuraron. En ese sentido, este artículo propone una aproximación a la dinámica en la provincia de Lima a través del análisis de la situación actual en las municipalidades distritales y de las condiciones en que adquirieron autonomía.

Este trabajo identificó que la dinámica en Lima-caracterizada por la transgresión a las entidades de gobierno local- ha sido determinada por la debilidad institucional, la falta de gobernabilidad y la ausencia de un sistema institucionalizado de partidos. Para los actores políticos locales, las municipalidades distritales representaron espacios de oportunidad para concentrar poder; debido a que la autonomía que tenían en sus jurisdicciones no era controlada por órganos con capacidad e independencia ni por un sistema de partidos institucionalizado que impidiera el uso del poder adquirido en función de intereses particulares de los incumbentes. Además, esta autonomía tampoco tuvo control por parte de la ciudadanía; ya que el interés de los electores se enfocaba principalmente en revertir el desequilibrio entre sus demandas sociales y la capacidad de las entidades de gobierno para atenderlas.

Palabras clave: gobernabilidad, autonomía, accountability, incumbente, Lima 


\begin{abstract}
Based on the media exposure of the number of authorities and ex-authorities prosecuted for corruption offenses at the regional and local level in 2014, interest in what was happening at these levels of government was resumed. Against this, the elimination of the possibility of immediate re-election in positions of popular election was taken as a measure. However, the problem around it requires an analysis of the dynamics that exist at these levels to understand what the factors that caused it were. In that sense, this article proposes an approximation to what happened in Lima through the analysis of the conditions in which the district municipalities acquired autonomy.

This article identified that the dynamics in Lima - characterized by the transgression of local government entities - has been shaped by institutional weakness, lack of governability and the absence of an institutionalized party system. For local political actors, the district municipalities represented opportunities to concentrate power, because the autonomy they had in their jurisdictions was not controlled by offices with capacity or by an institutionalized party system that prevented its use for particular interests of the incumbent. In addition, they didn't hold accountable, since the voters cared to revert the imbalance between their social demands and the capacity of the government entities to attend them.
\end{abstract}

Keywords: governability, autonomy, accountability, incumbent, Lima 


\section{Introducción}

El 2014 fue un año particularmente relevante para la atención a la corrupción en los tres niveles de Gobierno (nacional, regional y local). Los medios de comunicación informaron sobre funcionarios y servidores públicos investigados por presuntamente incurrir en delitos de corrupción y lavado de activos. Por un lado, se formalizó la denuncia penal contra un expresidente por un caso de lavado de activos en el Ministerio Público. (La República, 2019) Por otro lado, fueron expuestos los procesos por corrupción que atravesaban gobernadores regionales de los departamentos de Cajamarca, Loreto, Pasco, Tumbes, Áncash y Madre de Dios. Sobre el fenómeno a nivel local, Radio Programas del Perú (2014) presentó información registrada por la Procuraduría Pública Especializada en Delitos de Corrupción (PPEDC): de los 1841 alcaldes, 1699 estuvieron bajo investigación por corrupción (454 alcaldes provinciales y 1245 alcaldes distritales).

Esta difusión permitió que se retomara la atención hacia lo que ocurría en el interior del país. Sin embargo, la discusión en torno a la problemática se centró en la incidencia de la posibilidad de reelección inmediata sobre ello. Bajo el supuesto de que la posibilidad de reelegirse permitía a los gobernadores y a los alcaldes concentrar poder, la medida que se propuso en el Congreso fue la restricción de reelección inmediata de autoridades de elección popular en estos niveles. Así, las elecciones regionales y municipales en el 2014 fueron las últimas en que se permitió. El 10 de marzo del 2015 se publicó la Ley N³0305, Ley de reforma de los artículos $191^{\circ}, 194^{\circ}$ y $203^{\circ}$ de la Constitución Política del Perú sobre denominación y no reelección inmediata de autoridades de los gobiernos regionales y de los alcaldes.

A pesar de ello, la captura de autoridades no reelegidas por estar presuntamente involucradas en casos de corrupción mostró que este fenómeno no solo implicaba a quienes lograron la reelección. En el 2016, fue detenido un ex gobernador regional de Tumbes por colusión agravada. Esta exautoridad solo fue elegida en una ocasión. En el 2017, fue arrestado el alcalde provincial de Tocache por tener una acusación que lo señalaba por ser el presunto jefe de la organización criminal "los Duendes”. Esta autoridad tampoco tuvo reelección inmediata. Más adelante, el 31 de enero del 2018, fue detenido el alcalde de Santa Rosa, elegido en una sola oportunidad, por presuntamente haber liderado la organización criminal “los Malditos de Santa Rosa”. 
Estos casos permiten repensar la relación que se ha atribuido a la reelección inmediata de autoridades regionales y locales, y su involucramiento en procesos por corrupción y crimen organizado. Por un lado, debe considerarse que, en las últimas elecciones en que la reelección inmediata estuvo permitida (2014), solo el $11 \%$ de los alcaldes provinciales, el 17\% de los alcaldes distritales y el 16\% de los gobernadores regionales lograron ser reelegidos. (Incio \& Aragón 2014:19). Por otro lado, para analizar la posibilidad de transgresión en la administración pública en los espacios de gobierno regional y local, deben tomarse en cuenta factores como la ausencia de fiscalización (Muñoz, 2014) y la debilidad de los partidos políticos (Zavaleta, 2014).

$\mathrm{Al}$ respecto, existen investigaciones en las ciencias sociales que han abordado estos temas. Sin embargo, estas se han concentrado principalmente en los espacios a nivel regional (Barrenechea, 2010; Muñoz, 2014; Remy, 2010; Ruiz, 2016; Vera 2010). Frente a ello, este artículo propone una aproximación a lo que ocurre a nivel local a través del análisis de la situación en las municipalidades distritales de la provincia de Lima y de las condiciones en que estas entidades adquirieron autonomía. ${ }^{1}$ Para ello, se recabó información mediante la revisión de fuentes secundarias, la realización de solicitudes de información a la PPEDC y la sistematización de datos del portal del Jurado Nacional de Elecciones (Infogob).

Como resultado, este artículo plantea que la dinámica a nivel local en Lima caracterizada por la transgresión a sus entidades de gobierno distrital- se ha configurado por la debilidad institucional, la falta de gobernabilidad a nivel local y la ausencia de un sistema de partidos institucionalizado. Si bien la reelección fue una condición favorable para que las autoridades pudieran mantenerse en el cargo y, de esa manera, tuvieran la posibilidad de concentrar poder; los factores mencionados fueron los determinantes para que tuvieran la posibilidad de transgredir en las entidades a su cargo. En concordancia con ello, la estructura del artículo se divide en tres apartados. El primero expone los aspectos a considerar en los espacios a nivel subnacional en Perú. Posteriormente, el segundo explica cuáles fueron las condiciones en las que se configuró la dinámica en el nivel local. Finalmente, el tercero presenta el análisis de la situación en que se encuentran los distritos de Lima actualmente.

\section{Aspectos a considerar en el espacio subnacional}

\footnotetext{
${ }^{1}$ La selección del caso se sustenta en su ubicación dentro del departamento con mayor cantidad de casos de corrupción en trámite según la PPEDC (2018).
} 
Como parte de las reformas institucionales en los países latinoamericanos, tanto en los de estructura federal como unitaria, se impulsaron procesos de descentralización que ocasionaron el establecimiento de nuevas formas de distribución de poder. En consecuencia, los sistemas políticos se reconfiguraron. (Falleti, 2010) A nivel subnacional surgió una suerte de dinámica propia. (Dosek, 2015) Si bien cada jurisdicción subyace en condiciones dispuestas desde el gobierno central, las reglas de juego en cada circunscripción se configuran sobre la base de sus propias características.

\subsection{Lo subnacional en Perú: las condiciones del proceso de descentralización}

En Perú, el proceso de descentralización fue impulsado en 1980 y en el 2002. A partir de ello se configuraron tres niveles de gobierno en la división de la administración pública: nacional, regional y local. Así, se llegaron a conformar tres entidades de gobierno -gobierno regional, municipalidad provincial y municipalidad distrital- que disponían de autonomía política, económica y administrativa. Sin embargo, el factor clave para su autodeterminación (tener una dinámica propia) fue la ausencia de un control efectivo que regulara el poder que se adquirió mediante la transferencia de recursos y la autonomía que le fue otorgada para el ejercicio de funciones.

Este proceso se dio en periodos de inestabilidad política y debilidad institucional. Por un lado, el Estado no se encontraba lo suficientemente fuerte para designar funciones. No se contaba con un sistema de control efectivo que los fiscalice. Por otro lado, el sistema de partidos no estaba institucionalizado ni contaba con organizaciones políticas fuertes con presencia a nivel subnacional, pues hubo una desconexión entre los partidos nacionales y los actores políticos regionales y locales. (Tanaka, 2005) En consecuencia, las entidades de gobierno subnacional se convirtieron en espacios de oportunidad para concentrar poder, pues no hubo formas de control que sirvieran de contrapeso a la autonomía que adquirieron.

Muestra de ello es la situación de las entidades de gobierno regional y local. Según la PPEDC (2018), a diciembre del 2017 y del 2018, los gobiernos regionales, las municipalidades provinciales y las municipalidades distritales fueron las que concentraron la mayor cantidad de casos de corrupción en trámite. Dentro de ello, las municipalidades distritales fueron las entidades agraviadas con mayor número de casos 
registrados según los informes estadísticos presentados por la Procuraduría Pública Especializada en Delitos de Corrupción.

Tabla 1: Entidades agraviadas con mayor cantidad de casos de corrupción en el 2018

\begin{tabular}{|c|c|}
\hline Entidad agraviada & $\begin{array}{c}\text { Cantidad de } \\
\text { casos }\end{array}$ \\
\hline Municipalidades distritales & 10878 \\
\hline Municipalidades provinciales & 6665 \\
\hline Gobiernos regionales & 4465 \\
\hline Policía Nacional del Perú & 3882 \\
\hline Ministerios del Estado & 3102 \\
\hline Instituciones educativas (colegios de educación & \\
básica) & 2621 \\
\hline Universidades e institutos de educación superior & 977 \\
\hline Organismos autónomos & 914 \\
\hline Poder Judicial & 680 \\
\hline Ministerio Público & 531 \\
\hline Fuerzas Armadas & 235 \\
\hline Congreso de la República & 102 \\
\hline No se precisa / otros & 5707 \\
\hline
\end{tabular}

Fuente: PPEDC (2019)

\subsection{El espacio político a nivel subnacional}

A partir de 1990, con la elección de Ricardo Belmont como alcalde de la Municipalidad de Lima y de Alberto Fujimori como presidente de la República, se evidenció el ascenso de nuevos actores políticos (outsiders) frente a la crisis de legitimidad de los partidos nacionales. (Tanaka, 2005) En ese contexto se reconfiguraron las condiciones en el ámbito político. Las prácticas clientelares y personalistas se adaptaron a las nuevas reglas de juego en este espacio.

Los nuevos escenarios -que surgieron a partir de los impulsos al proceso de descentralización- impactaron en la política a nivel subnacional. Frente a la crisis de los partidos, surgieron movimientos regionales donde los actores centrales en el nivel regional y el local estuvieron desvinculados del espacio central, pero se unieron entre sí bajo la forma de coaliciones de independientes. (Zavaleta, 2014) “Los partidos locales no cuentan con incentivos que los motiven a formar parte de partidos nacionales, pues además de la mala reputación, estos no ofrecen nada a sus candidatos.” (De Gramont, 2010 citado en Muñoz, 2014, p. 4) 


\section{3.(In)estabilidad democrática a nivel subnacional}

En las condiciones de debilidad institucional y fragilidad del sistema de partidos que tenía Perú a nivel subnacional, la descentralización y democratización otorgaron un alto nivel de autonomía a los actores políticos regionales y locales. Por un lado, sin un órgano de control efectivo que regulara la capacidad administrativa, económica y política; las entidades de gobierno se convirtieron es espacios de oportunidad para obtener poder sin regulación.

Por otro lado, la falta de institucionalización de partidos permitió que se construyan liderazgos independientes sin que se les supervisara las prácticas políticas a las que pudieran recurrir ni las dinámicas que pudieran crear por la superposición de los intereses de los actores políticos. Los espacios de gobierno regional y local fueron vulnerables a que las prácticas clientelares y personalistas se trasladaran a ellas mediante el patronazgo. Las redes creadas en el espacio político tuvieron un escenario oportuno para desplazarse a la administración pública a través del otorgamiento de cargos.

En consecuencia, esta dinámica hizo posible que surgiera una suerte de organización paralela a la estatal: una institución informal que funcionaba al mismo tiempo que la entidad formal. (Helmke \& Levitsky, 2006) Los casos por corrupción y otros delitos como lavado de activos donde estuvieron presuntamente involucrados funcionarios y servidores públicos de estas entidades de gobierno muestran cómo la administración pública fue transgredida. Siguiendo a Francisco Durand (2012,2016), hubo un escenario oportuno para la captura del Estado.

Tabla 2: Exautoridades sentenciadas y procesadas a mayo del 2018

\begin{tabular}{|c|c|c|c|}
\hline Departamento & $\begin{array}{c}\text { Gobernador } \\
\text { regional }\end{array}$ & Alcalde provincial & Alcalde distrital \\
\hline Amazonas & 4 & 20 & 72 \\
\hline Ancash & 2 & 38 & 109 \\
\hline Apurímac & 4 & 9 & 61 \\
\hline Arequipa & 3 & 20 & 129 \\
\hline Ayacucho & 1 & 5 & 50 \\
\hline Cajamarca & 2 & 18 & 97 \\
\hline Callao & 1 & 1 & 5 \\
\hline
\end{tabular}




\begin{tabular}{|c|c|c|c|}
\hline Cusco & 5 & 38 & 160 \\
\hline Huancavelica & 2 & 7 & 67 \\
\hline Huánuco & 2 & 14 & 53 \\
\hline Ica & 1 & 14 & 64 \\
\hline Junín & 3 & 13 & 146 \\
\hline La Libertad & 2 & 10 & 49 \\
\hline Lambayeque & 1 & 7 & 63 \\
\hline Lima & 3 & 14 & 179 \\
\hline Loreto & 2 & 8 & 24 \\
\hline Madre De Dios & 3 & 7 & 21 \\
\hline Moquegua & 2 & 6 & 22 \\
\hline Pasco & 2 & 10 & 31 \\
\hline Piura & 3 & 21 & 54 \\
\hline Puno & 2 & 13 & 45 \\
\hline San Martín & 0 & 22 & 79 \\
\hline Tacna & 2 & 8 & 33 \\
\hline Tumbes & 3 & 13 & 19 \\
\hline Ucayali & 2 & 8 & 26 \\
\hline Total & 57 & 344 & 1658 \\
\hline
\end{tabular}

Fuente: PPEDC (2018)

Al 17 de diciembre del 2018 se expusieron en medios veintiún casos de alcaldes detenidos por estar comprendidos en investigaciones por delitos de corrupción, tráfico de terrenos, lavado de activos y otros, así como por presuntamente formar parte de organizaciones criminales. (El Comercio, 2018) A partir de ello, se muestra la transgresión a la administración pública en estos niveles de gobierno. "La estructura criminal por sus propias características, al conformarse al interior del sector público, facilita la comisión continuada y coordinada de los actos corruptos. Así, se perpetúan actividades ilícitas en el sector público con la ayuda de una diversidad de operarios no identificados y reemplazables.” (Solis y Puchuri 2019)

Tabla 3: Exautoridades sentenciadas a junio del 2018

\begin{tabular}{|c|c|c|c|}
\hline Departamento & $\begin{array}{c}\text { Gobernador } \\
\text { regional }\end{array}$ & $\begin{array}{c}\text { Alcalde } \\
\text { provincial }\end{array}$ & Alcalde distrital \\
\hline Amazonas & 1 & 2 & 12 \\
\hline Áncash & & 2 & 22 \\
\hline Apurímac & & 1 & 13 \\
\hline Arequipa & & & 4 \\
\hline Ayacucho & & & 9 \\
\hline Cajamarca & & 2 & 10 \\
\hline Cusco & 1 & 2 & 4 \\
\hline Huancavelica & & & 7 \\
\hline Huánuco & & & 2 \\
\hline Ica & & 1 & \\
\hline
\end{tabular}




\begin{tabular}{|c|c|c|c|} 
Junín & & & 6 \\
\hline La Libertad & & 1 & 2 \\
\hline Lambayeque & & 1 & 4 \\
\hline Lima & & & 14 \\
\hline Loreto & & & 2 \\
\hline Madre de Dios & & 1 & 2 \\
\hline Moquegua & & 1 & 8 \\
\hline Pasco & & & 2 \\
\hline Piura & & & 1 \\
\hline Puno & 1 & 1 & 8 \\
\hline San Martín & & 2 & 7 \\
\hline Tacna & & 2 & 3 \\
\hline Tumbes & & 3 & \\
\hline Ucayali & & & \\
\hline
\end{tabular}

Fuente:PPEDC (2018)

En estas condiciones, estos casos por delitos en agravio del Estado permiten reflexionar sobre las condiciones de democracia en estos niveles. (Stokes \&Amaral, 2005; Gibson, 2005) A pesar de ello, según Proética (2017), la tolerancia a la corrupción en la política es representada por un 48\%. Dentro de ello, la premisa "no se debe sancionar a funcionarios corruptos que hacen obras en beneficio de la población” se mantiene en la percepción de los ciudadanos sobre este fenómeno. Es decir, la transgresión que inicia en el espacio político y se traslada a la administración pública es, de alguna manera, aceptada por la población.

\section{Condiciones en las que se desarrolló la dinámica local}

El proceso de descentralización se desarrolló en periodos en los que las condiciones en el ámbito político e institucional no eran favorables para el otorgamiento de funciones a nivel subnacional. La ausencia de un aparato estatal fuerte y de partidos políticos institucionalizados favoreció que las entidades de gobierno subnacional tuvieran la posibilidad de convertirse en espacios de oportunidad para la concentración de poder.

Sin embargo, está situación es particular a nivel local, específicamente en las municipalidades distritales. Estas instancias recibieron autonomía administrativa, económica y política en condiciones en las que no hubo control efectivo a las entidades 
de gobierno. (Muñoz, 2014) Muestra de ello es la existencia de casos de corrupción y crimen organizado en las que exalcaldes distritales están presuntamente involucrados. Cabe resaltar que esa situación implicó a autoridades que solo tuvieron una gestión, a quienes fueron reelegidos de manera inmediata y a los que volvieron a ocupar el cargo después de uno o más periodos desde su último gobierno.

Tabla 4: Municipalidades provinciales y distritales con alcaldes investigados y/o procesados por casos de corrupción en trámite a junio del 2017

\begin{tabular}{|c|c|c|c|c|}
\hline Departamento & $\begin{array}{c}\text { Número de } \\
\text { municipalidades } \\
\text { provinciales } \\
\text { con alcaldes } \\
\text { investigados y/o } \\
\text { procesados }\end{array}$ & $\begin{array}{c}\text { Número de } \\
\text { municipalidades } \\
\text { provinciales } \\
\text { existentes }\end{array}$ & $\begin{array}{c}\text { Número de } \\
\text { municipalidades } \\
\text { distritales con } \\
\text { alcaldes } \\
\text { investigados y/o } \\
\text { procesados }\end{array}$ & $\begin{array}{c}\text { Número de } \\
\text { municipalidades } \\
\text { distritales } \\
\text { existentes }\end{array}$ \\
\hline Amazonas & 5 & 7 & 14 & 77 \\
\hline Áncash & 6 & 20 & 36 & 146 \\
\hline Apurímac & 2 & 7 & 6 & 74 \\
\hline Arequipa & 3 & 8 & 36 & 101 \\
\hline Ayacucho & 6 & 11 & 36 & 105 \\
\hline Cajamarca & 5 & 13 & 41 & 114 \\
\hline Callao & 1 & 1 & 3 & 6 \\
\hline Cusco & 6 & 13 & 26 & 97 \\
\hline Huancavelica & 3 & 7 & 11 & 90 \\
\hline Huánuco & 6 & 11 & 18 & 66 \\
\hline Ica & 3 & 5 & 9 & 38 \\
\hline Junín & 8 & 9 & 16 & 114 \\
\hline La Libertad & 7 & 12 & 29 & 71 \\
\hline Lambayeque & 3 & 3 & 14 & 35 \\
\hline Lima & 4 & 9 & 46 & 161 \\
\hline Loreto & 5 & 8 & 9 & 44 \\
\hline Madre de Dios & 2 & 3 & 4 & 8 \\
\hline Moquegua & 3 & 3 & 5 & 17 \\
\hline Pasco & 3 & 3 & 8 & 26 \\
\hline Piura & 6 & 8 & 18 & 57 \\
\hline Puno & 3 & 13 & 9 & 96 \\
\hline San Martín & 5 & 10 & 10 & 67 \\
\hline Tacna & 2 & 4 & 9 & 23 \\
\hline Tumbes & 1 & 3 & 5 & 10 \\
\hline Ucayali & 4 & 4 & 8 & 11 \\
\hline
\end{tabular}

Fuente: Plataforma Anticorrupción (2017) 


\subsection{La autonomía y el control en el nivel local}

La elección de autoridades locales fue básicamente por decisión del Ejecutivo desde 1919. Posteriormente, las condiciones cambiaron cuando la Constitución de 1979 dispuso la elección de autoridades locales y reconoció formalmente a las entidades de gobierno provincial y municipal. ${ }^{2}$ En consecuencia, se dieron cambios en el diseño y en la estructura institucional que permitieron la reconfiguración de este nivel de gobierno. Estas entidades de administración local fueron parte de una nueva distribución de funciones y transferencia de recursos.

Esta estructura se modificó nuevamente con la creación de un nivel intermedio de gestión: el gobierno regional. Dado que estas entidades también adquirieron autonomía administrativa, económica y política en condiciones de debilidad institucional y precariedad política, el alcance de los órganos de fiscalización y control se complejizó más. (Muñoz, 2014) En consecuencia, los gobiernos locales quedaron en una posición más alejada del gobierno central. Estas entidades se convirtieron en una suerte de espacio donde era posible mantenerse en el cargo (por la posibilidad de reelección inmediata) sin contrapesos.

La debilidad institucional en la capacidad de control a los gobiernos locales se evidenció con el limitado alcance de los órganos de control. Los órganos de control institucional no tenían la autonomía ni la capacidad suficiente para ejercer sus funciones sobre las entidades que supervisaban. Recién en el 2018 se aprobó la Ley N³0742, Ley de Fortalecimiento de la Contraloría General de la República y del Sistema Nacional de Control, mediante la cual se intentó impulsar la independencia (respecto de las entidades en que se encontraban) y la mejora de las condiciones de estas oficinas. ${ }^{3}$ Dentro de las disposiciones resaltó la incorporación progresiva de los órganos de control a la Contraloría General de la República.

\subsection{La relación entre los actores políticos locales y los partidos políticos nacionales}

\footnotetext{
${ }^{2}$ Cabe resaltar que en 1963 -durante el gobierno de Fernando Belaunde- se dispuso la realización de elecciones municipales.

3 Cabe resaltar que con esta ley se modificaron artículos de la Ley 27785 - Ley Orgánica del Sistema Nacional de Control y de la Contraloría General de la República.
} 
El espacio político en el nivel local estuvo caracterizado por el surgimiento de organizaciones políticas independientes frente a la pérdida de presencia de los partidos políticos nacionales. Con ello se configuraron nuevas formas de participación política. Los actores políticos locales tuvieron la oportunidad de conformar sus propias agrupaciones y crear liderazgos locales. De esta manera, se establecieron dinámicas particulares en los espacios políticos locales.

En relación con ello, cabe mencionar que Seifert (2014) identificó la tendencia inversa de los partidos políticos nacionales y de las organizaciones políticas independientes en las elecciones realizadas desde 1980 hasta las del 2010. Mientras el primer grupo fue reduciendo su presencia en los procesos electorales, el segundo aumentó su participación. (2014, p.29)

Tabla 5: Tipos de organizaciones políticas de candidatos elegidos en las elecciones municipales del 2002 y 2010

\begin{tabular}{|c|c|c|c|c|}
\hline \multirow{2}{*}{$\begin{array}{c}\text { Cargo } \\
\text { ostentado }\end{array}$} & $\begin{array}{c}|c| \\
\text { Partidos } \\
\text { nacionales }\end{array}$ & $\begin{array}{c}\text { Partidos } \\
\text { regionales }\end{array}$ & $\begin{array}{c}\text { Partidos } \\
\text { nacionales }\end{array}$ & $\begin{array}{c}\text { Partidos } \\
\text { regionales }\end{array}$ \\
\hline $\begin{array}{c}\text { Alcalde } \\
\text { provincial }\end{array}$ & $55 \%$ & $16 \%$ & $31 \%$ & $65 \%$ \\
\hline $\begin{array}{c}\text { Alcalde } \\
\text { distrital }\end{array}$ & $60 \%$ & $12 \%$ & $12 \%$ & $59 \%$ \\
\hline
\end{tabular}

Fuente: Seifert (2014, p.108)

\subsection{La posibilidad de reelección de autoridades a nivel local}

Los procesos electorales en el nivel local -elecciones para alcaldes provinciales y distritales- se han caracterizado por la intención de las autoridades por permanecer en el cargo a través de su presentación en los comicios: incumbencia. Sin embargo, un reducido porcentaje de estos lo logró. Como parte de esta situación, también se identificó que el rol de las organizaciones políticas fue básicamente instrumental, pues los candidatos solo lo utilizan para poder cumplir con el requisito de presentarse con una agrupación. De esta manera, la búsqueda de reelección se determinó por los actores políticos y no por las organizaciones políticas. (Incio y Aragón, 2014, p.16) 
Sobre la base de lo identificado por Incio y Aragón (2014) se reconoce que aunque más del $50 \%$ de los alcaldes provinciales y de los alcaldes distritales se presentaron y participaron en los comicios electorales, un porcentaje reducido lo logró. Asimismo, se identificó que en Piura, Lambayeque, Cajamarca, Loreto, Lima, Callao, Tumbes, San Martín y Lima Provincias se registró mayor incidencia de incumbencia por parte de los acaldes distritales en las elecciones del 2014. (2014, p.20)

Tabla 6: Porcentaje de los alcaldes que intentaron ser reelegidos y de los que fueron elegidos

\begin{tabular}{|c|c|c|c|c|}
\hline Año & $\begin{array}{c}\text { Porcentaje de } \\
\text { alcaldes } \\
\text { distritales que } \\
\text { intentaron ser } \\
\text { reelegidas }\end{array}$ & $\begin{array}{c}\text { Porcentaje de } \\
\text { alcaldes } \\
\text { distritales } \\
\text { reelectos }\end{array}$ & $\begin{array}{c}\text { Porcentaje de } \\
\text { alcaldes } \\
\text { provinciales } \\
\text { que intentaron } \\
\text { ser reelegidas }\end{array}$ & $\begin{array}{c}\text { Porcentaje } \\
\text { de alcaldes } \\
\text { provinciales } \\
\text { reelectos }\end{array}$ \\
\hline 2006 & $62.15 \%$ & $35.27 \%$ & $57.43 \%$ & $22.32 \%$ \\
\hline 2010 & $56.53 \%$ & $33.59 \%$ & $58.97 \%$ & $27.83 \%$ \\
\hline 2014 & $60.62 \%$ & $17.41 \%$ & $52.31 \%$ & $10.77 \%$ \\
\hline
\end{tabular}

Fuente: Elaboración propia sobre la base de Incio \& Aragón (2014, p.19)

\section{La dinámica en los distritos de Lima}

Al igual que otras ciudades del interior del país, Lima se pobló producto de la migración. Sin embargo, su ubicación como capital del país ocasionó que la mayoría de migrantes se reubicaran en sus distritos. La mayoría de ellos ocupó las zonas marginales principalmente. Esto provocó una suerte de desborde de la capacidad que tenían los distritos existentes hasta ese momento. (Matos Mar, 2004) Así, se configuraron los distritos urbanos marginales donde las personas crearon una suerte dinámica propia adaptando lo ya establecido en el lugar: las prácticas sociales limeñas se reconfiguraron (Huerta, 2012)

Estas nuevas condiciones influyeron en la reconfiguración de las prácticas de clientelismo y patronazgo en el espacio político. Los migrantes se convirtieron en un nuevo grupo al que considerar en este ámbito. Tanto desde el gobierno central como desde el local se empezaron a atender sus demandas. Estas se centraron en tres aspectos principalmente: vivienda, transporte y comercio. Al mismo tiempo, el proceso de descentralización permitió que las municipalidades distritales adquirieran mayor autonomía para el ejercicio de funciones y la disposición de recursos. En consecuencia, 
la dinámica local en los distritos de Lima se caracterizó por la convergencia de los cambios en los aspectos social, político e institucional.

\subsection{La entidad de gobierno municipal en los distritos de Lima}

La mayoría de circunscripciones periféricas fueron pobladas por migrantes provenientes del interior del país que se instalaron principalmente invadiendo terrenos. Estas se convirtieron en distritos urbano-marginales donde la ocupación territorial fue desordenada. Asimismo, como parte de la adaptación de los migrantes a los distritos limeños, la informalidad se convirtió en la principal manera de desarrollar sus actividades. (Aramburú et al. 2004) Por un lado, las personas utilizaron un transporte alternativo para movilizarse en los cerros que poblaron: el mototaxi. También se recurrió a otros medios: los taxis colectivos y las minivan. Por otro lado, se recurrió a la venta ambulante como actividad económica. (De Soto 1986)

De esta manera, los distritos de Lima tuvieron cuatro principales características: la informalidad, la población migrante, la pobreza y la necesidad de urbanización. Cada una de ellas representó un aspecto sobre el cual la población tenía demandas. No obstante, estas se concentraron principalmente en dos aspectos. Por un lado, los sectores pobres necesitaban programas sociales. Por otro lado, frente a la falta de urbanización se exigieron obras públicas. En esas condiciones, los incumbentes tenían un escenario oportuno para obtener apoyo popular si las atendían.

Gráfico 1: Determinación de demandas locales a partir de las características de los distritos

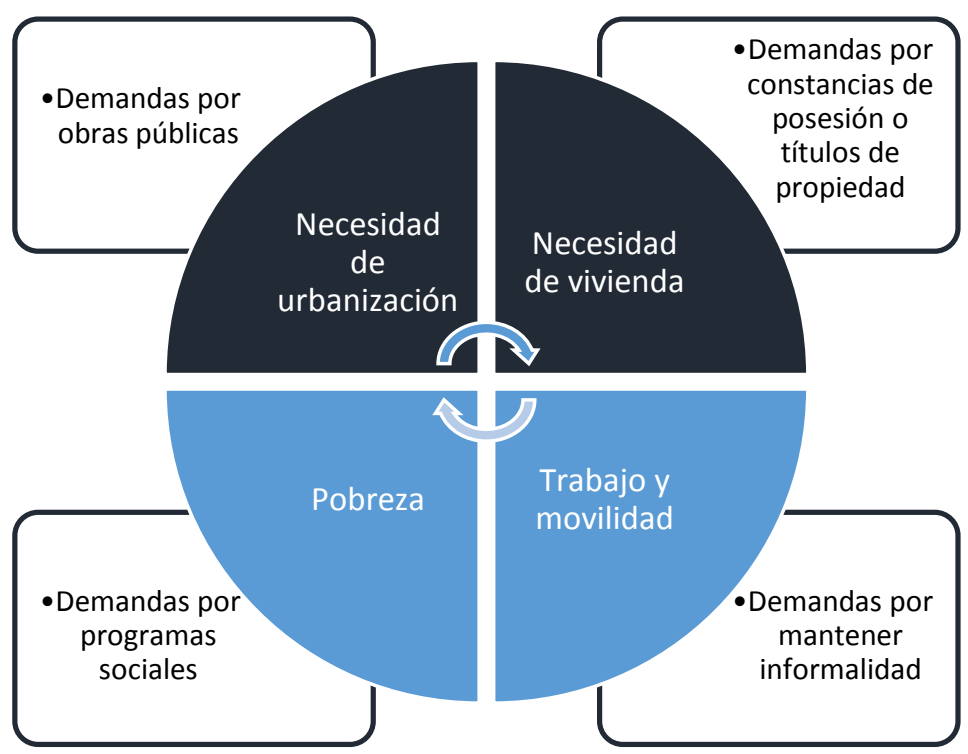


Fuente: Elaboración propia sobre la base de Aramburú et al.(2004), De Soto (1986) y Matos Mar(2004)

Esta nueva situación en los distritos de Lima tuvo un impacto en el aspecto político. Los nuevos grupos se constituyeron como sectores políticos. Establecieron la atención a sus demandas (en función a sus intereses y condiciones) como condición para que los actores políticos obtengan apoyo popular. "La historia de la urbanización de Lima los últimos cuarenta años está marcada por los procesos de migración, invasión de terrenos y la negociación/confrontación con el Estado”. (Aramburú et al., 2004, p.30) Esta dinámica muestra que para estos sectores lo más importante era la gobernabilidad (la atención de sus demandas por parte del sistema político). ${ }^{4}$

$\mathrm{Al}$ respecto, cabe señalar que los procesos de gestión -mediante los que se atienden uno de los aspectos más demandados por parte de los electores- son los mismos donde se concentra la mayor cantidad de casos de corrupción. Según la PPEDC (2018), a mayo del 2018, el 58\% de los casos de corrupción en trámite donde estuvieron presuntamente involucradas autoridades regionales y locales (elegidas entre el 2002 y 2014) se encontraron vinculados a procesos de contratación. Dentro de ellos, el 53\% estuvo relacionado con obras y el 47\%, a bienes y servicios. Asimismo, en el mismo grupo, se reportó que en el 51\% de los casos la etapa más recurrente fue la fase de ejecución contractual; el 28\%, en actos preparatorios; y el 21\%, en los procesos de selección.

\section{Tabla 7: Defraudación al Estado en las contrataciones por fases según la PPEDC ${ }^{5}$}

\begin{tabular}{|c|c|}
\hline Fase & Materialización de defraudación \\
\hline & $\begin{array}{l}\text { - Financiamiento y/o liquidez ilegal a favor del contratista, mediante } \\
\text { adelantos y/o pagos indebidos a pesar de que la prestación aún no se } \\
\text { ha ejecutado y/o culminado. } \\
\text { - Liberar al contratista de la parte más compleja de la obra y/o } \\
\text { servicio y/o bien en menoscabo para el Estado, a través de } \\
\text { reducciones ilegales. } \\
\text { Mayor costos a la presentación a causa de (1) ampliaciones de } \\
\text { plazos simulados y/o aprobados ilegalmente, con la finalidad de } \\
\text { evadir la aplicación de penalidades y/o generar el pago al contratista } \\
\text { de mayores gastos generales; (2) modificaciones al contrato } \\
\text { simuladas y/o aprobadas ilegalmente; y (3) adicionales simulados } \\
\text { y/o aprobados ilegalmente. }\end{array}$ \\
\hline sto & ionarios \\
\hline
\end{tabular}

\footnotetext{
${ }^{4}$ En este trabajo se entiende la gobernabilidad como "el equilibrio dinámico entre las demandas sociales y capacidad del sistema político de atención y respuesta legítima y eficaz". (Camou, 2001, p.36)

${ }^{5}$ Esta es una clasificación referencial presentada por la PPEDC (2018) a partir de los casos analizados.
} 


\begin{tabular}{|l|l|}
\hline preparatorios & $\begin{array}{l}\text { gobiernos regionales y locales antes de que se inicie el proceso de } \\
\text { selección con el fin de direccionar el otorgamiento de la buena pro o } \\
\text { que se permitan los actos de defraudación antes identificados } \\
\text { durante la fase de ejecución contractual. } \\
\text { - El favorecimiento a proveedores con quienes se contrata a pesar de } \\
\text { que están impedidos de ser participantes, postores, contratistas y/o } \\
\text { subcontratistas. }\end{array}$ \\
\hline
\end{tabular}

Fuente: PPEDC (2018)

A partir de lo mostrado se puede comprender cómo la falta de gobernabilidad influyó en la configuración de una dinámica local caracterizada por la transgresión a las entidades de gobierno de ese nivel. La preocupación de estos sectores por la atención a sus demandas -pese a que las autoridades pueden incurrir en delitos de corrupción en ese ámbito- permite comprender por qué el $41 \%$ de los encuestados por Datum Internacional en Lima en el 2014 afirmó que votaría por un candidato que robe siempre y cuando haga obras. (Meléndez, 2014) En la misma línea, esto explica, de alguna manera, la reelección de alcaldes, pese a estar comprendidos en investigaciones por delitos como corrupción.

\subsection{El rol de las organizaciones políticas}

Hasta las elecciones regionales y municipales del 2014, las autoridades podían presentarse en los comicios para lograr la reelección inmediata. A pesar de ello, un reducido grupo lo logró. En las elecciones de los distritos de Lima, comprendidos entre 1963 y 2014, 90 alcaldes han tenido gestiones consecutivas mediante el logro de reelecciones inmediatas y en 22 casos lograron volver al cargo después de al menos un periodo de gestión. Dentro del grupo de los alcaldes distritales que fueron elegidos de manera inmediata, solo cinco lograron más de cuatro gobiernos consecutivos y el $80 \%$ consiguió mantener solo dos gestiones consecutivas.

Tabla 8: Reelecciones consecutivas en los distritos de Lima desde 1963 hasta el $2018^{6}$

\begin{tabular}{|c|c|}
\hline $\begin{array}{c}\text { Número de reelecciones } \\
\text { consecutivas }\end{array}$ & $\begin{array}{c}\text { Número de alcaldes reelegidos } \\
\text { de manera inmediata }\end{array}$ \\
\hline 1 & 66 \\
\hline 2 & 12 \\
\hline 3 & 3 \\
\hline 4 & 3 \\
\hline
\end{tabular}

\footnotetext{
${ }^{6}$ El cuadro no considera los casos particulares de alcaldes que fueron elegidos después de conseguir una relección inmediata ni los que fueron reelegidos de manera no inmediata.
} 


\begin{tabular}{|c|c|}
\hline 5 & 0 \\
\hline 6 & 2 \\
\hline
\end{tabular}

Fuente: Elaboración propia sobre la base de información del portal Infogob

Tabla 9: Casos particulares de reelección en Lima

\begin{tabular}{|c|c|c|}
\hline Distrito & $\begin{array}{l}\text { Descripción de casos de } \\
\text { alcaldes reelegidos }\end{array}$ & Años de elecciones ${ }^{7}$ \\
\hline Ancón & $\begin{array}{l}\text { El alcalde fue elegido en las } \\
\text { elecciones de 1989, } 1993 \text { y } \\
1998 .\end{array}$ & $1989-1993-1995-1998$ \\
\hline Ate & $\begin{array}{l}\text { El alcalde fue elegido en las } \\
\text { elecciones de 1998, 2002, } \\
2010 \text { y } 2014 .\end{array}$ & 1998-2002-2006-2010-2014 \\
\hline Barranco & $\begin{array}{l}\text { El alcalde fue elegido en las } \\
\text { elecciones del } 2006 \text { y del } \\
2014 \text {. }\end{array}$ & $2006-2010-2014$ \\
\hline Breña & $\begin{array}{l}\text { El alcalde fue elegido en los } \\
\text { comicios de 1989, } 1993 \text { y } \\
1998 .\end{array}$ & 1963-1966-1980-1983-1986-1989-1993-1995 \\
\hline Chorrillos & $\begin{array}{l}\text { El alcalde fue elegido en las } \\
\text { elecciones de 1980, 1983, } \\
1989 \text { y } 1995 .\end{array}$ & $1980-1983-1986-1989-1993-1995$ \\
\hline Cieneguilla & $\begin{array}{l}\text { El alcalde fue elegido en las } \\
\text { elecciones de } 1980 \text { y } 1989 .\end{array}$ & 1980-1983-1986-1989 \\
\hline \multirow{2}{*}{ Comas } & $\begin{array}{l}\text { El alcalde fue elegido en las } \\
\text { elecciones de 1980, 1983, } \\
1986 \text { y } 1998 .\end{array}$ & 1980-1983-1986-1989-1993-1995-1998 \\
\hline & $\begin{array}{l}\text { El alcalde fue elegido en las } \\
\text { elecciones del 2002, } 2006 \text { y } \\
2014 \text {. }\end{array}$ & $2002-2006-2010-2014$ \\
\hline Jesús María & $\begin{array}{l}\text { El alcalde fue elegido en las } \\
\text { elecciones del } 2002 \text { y } 2014 .\end{array}$ & $2002-2006-2010-2014$ \\
\hline La Victoria & $\begin{array}{l}\text { El alcalde fue elegido en las } \\
\text { elecciones de } 1983 \text { y } 2002 .\end{array}$ & 1983-1986-1989-1993-1995-1998-2002 \\
\hline Lima & $\begin{array}{l}\text { El alcalde fue elegido en las } \\
\text { elecciones 2002, } 2006 \quad \text { y } \\
2014 \text {. }\end{array}$ & $2002-2006-2010-2014$ \\
\hline \multirow{2}{*}{ Miraflores } & $\begin{array}{l}\text { El alcalde fue elegido en las } \\
\text { elecciones de 1983, } 1986 \text { y } \\
1998 .\end{array}$ & 1983-1986-1989-1993-1995-1998 \\
\hline & $\begin{array}{l}\text { El alcalde fue elegido en las } \\
\text { elecciones de } 1995 \text { y en el } \\
2002 \text {. }\end{array}$ & 1995-1998-2002 \\
\hline Pachacamac & $\begin{array}{l}\text { El alcalde fue elegido en las } \\
\text { elecciones de } 1963 \text { y } 1983 .\end{array}$ & $1963-1966-1980-1983$ \\
\hline Pucusana & $\begin{array}{l}\text { El alcalde fue elegido en las } \\
\text { elecciones de } 1963,1980 \text { y }\end{array}$ & $1963-1966-1980-1983$ \\
\hline
\end{tabular}

${ }^{7}$ A partir de 1998, se convocó a elecciones municipales cada cuatro años. 


\begin{tabular}{|c|c|c|}
\hline & 1983. & \\
\hline & $\begin{array}{l}\text { El alcalde fue elegido en las } \\
\text { elecciones de 1998, } 2010 \text { y } \\
2014 \text {. }\end{array}$ & 1998-2002-2006-2010-2014 \\
\hline Punta Negra & $\begin{array}{l}\text { El alcalde fue elegido en las } \\
\text { elecciones de 1963, } 1966 \text { y } \\
1983 .\end{array}$ & $1963-1966-1980-1983$ \\
\hline $\begin{array}{l}\text { San Juan de } \\
\text { Miraflores }\end{array}$ & $\begin{array}{l}\text { El alcalde fue elegido en las } \\
\text { elecciones de 1983, 1986, } \\
1995 \text { y } 1998 .\end{array}$ & 1983-1986-1989-1993-1995-1998 \\
\hline San Luis & $\begin{array}{l}\text { El alcalde fue elegido en las } \\
\text { elecciones de } 1993 \text { y } 1998 .\end{array}$ & $1993-1995-1998$ \\
\hline Santa Anita & $\begin{array}{l}\text { El alcalde fue elegido en las } \\
\text { elecciones de 1995, } 1998 \text { y } \\
2006 \text {. }\end{array}$ & 1995-1998-2002-2006 \\
\hline Surquillo & $\begin{array}{l}\text { El alcalde fue elegido en las } \\
\text { elecciones de 1989, } 1993 \text { y } \\
1998 .\end{array}$ & 1989-1993-1995-1998 \\
\hline $\begin{array}{l}\text { Villa El } \\
\text { Salvador }\end{array}$ & $\begin{array}{l}\text { El alcalde fue elegido en las } \\
\text { elecciones de 1983, } 1986 \text { y } \\
1995 .\end{array}$ & $1983-1986-1993-1995$ \\
\hline
\end{tabular}

Fuente: Elaboración propia sobre la base de información del portal Infogob

Asimismo, a partir del análisis de los resultados electorales en los distritos de Lima se identifica una tendencia según la cual en la mayoría de las jurisdicciones distritales se eligieron a candidatos que se presentaban con la misma agrupación que el ganador en Lima. Aunque también se reconoce que surgieron diversas organizaciones políticas locales como alternativas a los partidos políticos tradicionales, esta relación entre los ganadores sugiere que a nivel local hubo algún tipo de relación entre los candidatos a Lima y los de otros distritos.

Tabla 10: Tendencia de las elecciones municipales en Lima

\begin{tabular}{|c|c|c|c|c|}
\hline \multirow{2}{*}{ AÑ } & \multicolumn{4}{|c|}{$\begin{array}{c}\text { Organizaciones políticas de los candidatos elegidos } \\
\text { en la mayoría de distritos de lima }\end{array}$} \\
\hline 1963 & $\begin{array}{c}\text { Alianza Acción Popular - } \\
\text { Democracia Cristiana }\end{array}$ & 15 & APRA - UNO & 13 \\
\hline 1966 & $\begin{array}{c}\text { Alianza Acción Popular - } \\
\text { Democracia Cristiana }\end{array}$ & 17 & APRA-UNO & 14 \\
\hline 1980 & Acción Popular & 23 & \\
\hline 1983 & Izquierda Unida & 18 & \\
\hline 1986 & Partido Aprista Peruano & 29 \\
\hline 1989 & Fredemo & 21 \\
\hline 1993 & Movimiento Obras & \\
\hline
\end{tabular}




\begin{tabular}{|c|c|c|c|c|}
\hline 1995 & Somos Lima & 20 & Cambio 90 & 19 \\
\hline 1998 & $\begin{array}{c}\text { Movimiento Independiente } \\
\text { Vamos Perú }\end{array}$ & 24 & $\begin{array}{c}\text { Movimiento Independiente } \\
\text { Vamos Vecino }\end{array}$ & 15 \\
\hline 2002 & Partido Democrático Somos Perú & 17 & $\begin{array}{c}\text { Alianza Electoral Unidad } \\
\text { Nacional }\end{array}$ & 16 \\
\hline 2006 & Alianza Electoral Unidad Nacional & 28 & 7 \\
\hline 2010 & Partido Popular Cristiano & 11 & Cambio Radical & 19 \\
\hline 2014 & \multicolumn{2}{|c|}{ Solidaridad Nacional } & \multicolumn{2}{c|}{19} \\
\hline
\end{tabular}

Fuente: Elaboración propia sobre la base de información del portal Infogob

Tabla 11: Organizaciones políticas elegidas en Lima y sus distritos

\begin{tabular}{|c|c|c|}
\hline $\begin{array}{l}\text { Año de } \\
\text { elección }\end{array}$ & $\begin{array}{l}\text { Organización elegida de los } \\
\text { candidatos elegidos en la } \\
\text { mayoría de distritos }\end{array}$ & $\begin{array}{l}\text { Organización política de candidato } \\
\text { elegido en Lima }\end{array}$ \\
\hline 1963 & $\begin{array}{c}\text { Alianza Acción Popular - } \\
\text { Democracia Cristiana }\end{array}$ & $\begin{array}{c}\text { Alianza Acción Popular - Democracia } \\
\text { Cristiana } \\
\end{array}$ \\
\hline 1966 & $\begin{array}{c}\text { Alianza Acción Popular - } \\
\text { Democracia Cristiana } \\
\end{array}$ & $\begin{array}{c}\text { Alianza Acción Popular - Democracia } \\
\text { Cristiana } \\
\end{array}$ \\
\hline 1980 & Acción Popular & Acción Popular \\
\hline 1983 & Izquierda Unida & Izquierda Unida \\
\hline 1986 & Izquierda Unida & Izquierda Unida \\
\hline 1989 & Fredemo & $\begin{array}{c}\text { Lista Independiente } \mathrm{N}^{0} 3 \text { Movimiento } \\
\text { Obras }\end{array}$ \\
\hline 1993 & Movimiento Obras & Movimiento Obras \\
\hline 1995 & Somos Lima & Somos Lima \\
\hline 1998 & $\begin{array}{l}\text { Movimiento Independiente } \\
\text { Vamos Perú }\end{array}$ & Movimiento Independiente Somos Perú \\
\hline 2002 & $\begin{array}{l}\text { Partido Democrático Somos } \\
\text { Perú }\end{array}$ & Alianza Electoral Unidad Nacional \\
\hline 2006 & $\begin{array}{l}\text { Alianza Electoral Unidad } \\
\text { Nacional }\end{array}$ & Alianza Electoral Unidad Nacional \\
\hline 2010 & PPC & Partido Descentralista Fuerza Social \\
\hline 2014 & Solidaridad Nacional & Solidaridad Nacional \\
\hline
\end{tabular}

Fuente: Elaboración propia sobre la base de información del portal Infogob

Sin embargo, esta situación no refleja un espacio político organizado a nivel local, sino el uso de las organizaciones políticas para presentarse a elecciones. Los candidatos de los distritos de Lima buscaron presentarse en sus respectivas jurisdicciones con las agrupaciones de quienes tenían mayor popularidad para la Municipalidad de Lima. Por consiguiente, los resultados electorales en los distritos de Lima muestran una dinámica 
política y no partidaria, tal como lo señalan Incio y Aragón para la situación a nivel subnacional (2014).

Tabla 12: Volatilidad en las elecciones distritales de 2002, 2006, 2010 y 2014 en

\section{Lima $^{8}$}

\begin{tabular}{|c|c|c|}
\hline Distritos & $\begin{array}{c}\text { Número de } \\
\text { alcaldes reelegidos }\end{array}$ & $\begin{array}{c}\text { Número de organizaciones políticas } \\
\text { por las que se presentaron }\end{array}$ \\
\hline Ancón & 0 & 0 \\
\hline Ate & 0 & 0 \\
\hline Barranco & 1 & 2 \\
\hline Breña & 1 & 1 \\
\hline Carabayllo & 2 & $\begin{array}{l}\text { Cada uno se presentó con una } \\
\text { organización política }\end{array}$ \\
\hline Chaclacayo & 1 & 2 \\
\hline Chorrillos & 1 & 2 \\
\hline Cieneguilla & 2 & $\begin{array}{l}\text { Uno se presentó con dos organizaciones } \\
\text { políticas y otro postuló solo con una. }\end{array}$ \\
\hline Comas & 1 & 2 \\
\hline El Agustino & 1 & 2 \\
\hline Independencia & 1 & 2 \\
\hline Jesús María & 2 & $\begin{array}{l}\text { Cada uno se presentó con dos } \\
\text { organizaciones políticas }\end{array}$ \\
\hline La Molina & 2 & $\begin{array}{l}\text { Cada uno se presentó con una sola } \\
\text { organización política }\end{array}$ \\
\hline La Victoria & 1 & 2 \\
\hline Lima & 1 & 2 \\
\hline Lince & 1 & 3 \\
\hline Los Olivos & 1 & 2 \\
\hline Lurigancho & 1 & 4 \\
\hline Lurín & 1 & 1 \\
\hline $\begin{array}{l}\text { Magdalena del } \\
\text { Mar }\end{array}$ & 1 & 2 \\
\hline Miraflores & 1 & 1 \\
\hline Pachacamac & 1 & 3 \\
\hline Pucusana & 1 & 2 \\
\hline Pueblo Libre & 1 & 2 \\
\hline Puente Piedra & 1 & 1 \\
\hline Punta Hermosa & 1 & 3 \\
\hline Punta Negra & 1 & 2 \\
\hline Rímac & 1 & 2 \\
\hline San Bartolo & 1 & 4 \\
\hline San Borja & 2 & $\begin{array}{c}\text { Cada uno se presentó con dos } \\
\text { organizaciones políticas. }\end{array}$ \\
\hline
\end{tabular}

\footnotetext{
${ }^{8}$ En este análisis solo se considera las organizaciones políticas con que las autoridades reelectas se hayan presentado desde el 2002 hasta el 2014. No toma en cuenta si alguno de ellos postuló con otras agrupaciones en anteriores comicios.
} 


\begin{tabular}{|c|c|c|}
\hline San Isidro & 0 & 0 \\
\hline $\begin{array}{c}\text { San Juan de } \\
\text { Lurigancho }\end{array}$ & 1 & 2 \\
\hline $\begin{array}{c}\text { San Juan de } \\
\text { Miraflores }\end{array}$ & 0 & 2 \\
\hline San Luis & 1 & 2 \\
\hline $\begin{array}{c}\text { San Martín de } \\
\text { Porres }\end{array}$ & 1 & 2 \\
\hline San Miguel & 1 & 2 \\
\hline Santa Anita & 1 & 2 \\
\hline $\begin{array}{c}\text { Santa María del } \\
\text { Mar }\end{array}$ & 1 & 2 \\
\hline Santa Rosa & 1 & 1 \\
\hline Santiago de Surco & 1 & 2 \\
\hline Surquillo & 2 & 0 \\
\hline Villa El Salvador & 1 & Cada uno se presentó con una \\
\hline Villa María del \\
Triunfo
\end{tabular}

Fuente: Elaboración propia sobre la base de información del portal Infogob

El impacto de esta ausencia de un sistema de partidos institucionalizado se evidencia en la existencia de investigaciones donde están comprendidos exalcaldes por presuntamente transgredir en la administración pública. La infiltración de presuntos miembros de organizaciones criminales y la incidencia en delitos de corrupción muestra la superposición de los intereses de actores políticos. Situación que en un sistema de partidos políticos institucionalizado se limitaría; pues los partidos políticos serían los que tienen el peso, la estabilidad y la legitimidad, en lugar de los actores políticos. (Huntington, 2006)

\subsection{La situación de los distritos de Lima al año 2018}

A partir de lo descrito se reconocen tres condiciones en las que se configuraron las dinámicas en los distritos de Lima. En primer lugar, no hubo un sistema de control efectivo que regulara el alcance de la autonomía que las municipalidades distritales adquirieron en lo administrativo, económico y político. En segundo lugar, el interés de la ciudadanía por lograr gobernabilidad (la atención a las necesidades de los electores) se imponía frente a otros aspectos como el control a los riesgos de transgresión en las entidades de gobierno local. En tercer lugar, la ausencia de un sistema de partidos institucionalizado favoreció que los intereses particulares de los 
políticos locales determinaran las prácticas políticas en los espacios políticos locales.

Sobre la base de ello, se comprende la existencia de alcaldes procesados y sentenciados por estar presuntamente involucrados en casos de corrupción en Lima. En esas condiciones de precariedad política, debilidad institucional y ausencia de gobernabilidad se favoreció la configuración de escenarios de oportunidad para que funcionarios y servidores públicos de esta entidad puedan transgredir en la administración pública. A octubre del 2018, de las autoridades distritales elegidas para los últimos cuatro periodos de gobierno en Lima (2002, 2006, 2010 y 2014), 63 se encuentran con casos de corrupción en trámite.

A partir del informe de la PPEDC (2018) se reconoce que en el departamento de Lima hubo siete alcaldes distritales sentenciados por delitos de corrupción. Dentro de ellos, cinco gobernaron en distritos de la provincia de Lima. En este grupo, dos tuvieron una sola gestión en su jurisdicción y tres lograron reelecciones consecutivas. Por consiguiente, esta información muestra que no solo las autoridades reelegidas de manera inmediata pudieron estar comprendidas en investigaciones por presunta transgresión a la administración pública. ${ }^{9}$

En el 2018, cinco exalcaldes de los distritos de la provincia de Lima fueron arrestados por estar comprendidos en investigaciones que se seguían a organizaciones criminales. ${ }^{10}$ De este grupo, cuatro se encontraban ocupando por primera vez el cargo. Solo uno de ellos estuvo por periodos de gobierno consecutivos. De esta manera, este panorama muestra que no fue necesario que las autoridades sean reelegidas para que logren una concentración de poder y transgredan en la administración pública.

\section{Conclusiones}

Este trabajo identificó que la dinámica a nivel local en Lima -caracterizada por la transgresión a sus entidades de gobierno distrital- se ha configurado por la debilidad

\footnotetext{
${ }^{9}$ Ya sea por delitos de corrupción, tráfico de terrenos u otros.

${ }^{10}$ Información recabada a partir del informe de la PPEDC (2018) y medios de comunicación
} 
institucional, la falta de gobernabilidad y la ausencia de un sistema de partidos institucionalizado. Para los actores políticos locales, las municipalidades distritales representaron espacios de oportunidad para concentrar poder; debido a que la autonomía en sus jurisdicciones no era controlada por órganos con capacidad e independencia ni por un sistema de partidos institucionalizado que impidiera el uso del poder adquirido para satisfacer intereses particulares de los incumbentes. Además, esta autonomía tampoco tenía contrapesos por parte de la ciudadanía, ya que a los electores les interesaba principalmente revertir el desequilibrio entre sus demandas sociales y la capacidad de las entidades de gobierno para atenderlas.

\section{Conclusiones específicas}

En primer lugar, la autonomía administrativa, política y económica otorgada a los gobiernos locales -a las municipalidades distritales de Lima en este caso- en contextos de debilidad institucional favoreció que las gestiones se convirtieran en una suerte de periodos de discrecionalidad para el ejercicio de sus funciones. Antes de la aprobación de la Ley $\mathrm{N}^{\circ} 30742$, los órganos de control institucional no tenían capacidad ni autonomía para supervisarlas, por lo que existía un escenario oportuno para tener la posibilidad de transgredir.

En segundo lugar, la falta de institucionalización de las organizaciones políticas favoreció que se formen políticos locales con poder y sin contrapesos. La satisfacción de los intereses de los actores políticos locales se pudo materializar una vez que obtuvieron el cargo. De esta manera, se comprende la existencia de investigaciones que comprendieron a exalcaldes por presunta relación con organizaciones criminales e incidencia en delitos de corrupción.

En tercer lugar, la ausencia de gobernabilidad influyó en la creación de escenarios oportunos para la posibilidad de concentración de poder y de transgresión en las entidades de gobierno. La atención de los electores a la política y la administración local se centró en la búsqueda de respuestas a sus demandas (programas sociales y obras públicas principalmente) y de la adaptación de las gestiones a las características de los 
distritos (la informalidad en la mayor parte de los casos), mas no en la posibilidad de que en esos ámbitos se pudiera incurrir en actos de corrupción.

En cuarto lugar, si bien la posibilidad de reelección inmediata permitió que los alcaldes pudieran concentrar poder, la existencia casos de incumbentes que solo lograron tener una gestión y durante ella fueron capturados por estar presuntamente involucrados en organizaciones criminales muestra que la debilidad institucional, la precariedad política y la ausencia de gobernabilidad fueron las condiciones que determinaron los escenarios de oportunidad para la configuración de dinámicas de transgresión a las entidades a su cargo. La posibilidad de cometer actos delictivos dentro de la administración pública la tuvieron tanto quienes fueron elegidos una vez como los que ocuparon más veces el cargo. 


\section{Bibliografía}

Alerta informativa (2014) Portal de Alerta informativa Recuperado de http://alertainformativa.blogspot.pe/ [Consulta: 15 de mayo del 2019]

Amaral, S., \& Stokes, S. (2005). Democracia local: clientelismo, capital social e innovación política en Argentina. Buenos Aires, Argentina: Editorial de la Universidad Nacional de Tres de Febrero.

Aula Abierta (productora). (2012). Aula abierta con Alex Huerta [videograbación]. Lima: Pontificia Universidad Católica del Perú. Recuperado de $\quad$ https://videos.pucp.edu.pe/videos/ver/2f8a6c5174e1eeaf83205aa12fa5cb3f [Consulta: 15 de mayo del 2019]

Aragón, J. \& Incio, J. (2014) La reelección de autoridades regionales y municipales en el Perú 2006-2014. Revista Argumentos, (5), 16-30. Recuperado de $\quad$ https://revistaargumentos.iep.org.pe/wpcontent/uploads/2014/12/aragonincio_dic14.pdf

Aramburú, C. et al. (2004) Perú hoy: las ciudades en el Perú. Lima: DESCO.

Barrenechea, R. (2014). Becas, bases y votos: Alianza para el Progreso y la política subnacional en el Perú. Lima, Perú: Instituto de Estudios Peruanos

Bazo, A. (2015) Actuales autoridades regionales y municipales no podrán reelegirse el 2018. La Ley. Recuperado de https://laley.pe/art/2273/actuales-autoridadesregionales-y-municipales-no-podran-reelegirse-el-2018 [Consulta: 15 de mayo del 2019]

Becerra, M.G. \& Aragón, J. (2016) El estudio de la política subnacional en el Perú: posibilidades, avances y desafíos. Lima: Pontificia Universidad Católica del Perú. Recuperado de file://C:/Users/Toshiba/Downloads/19201-76292-1-PB.pdf

Briceño, A. (2018) Elías Cuba: organización criminal empezó con la campaña del 2014, $\begin{array}{lllll}\text { afirma } & \text { fiscal. } & \text { El } & \text { Comercio. }\end{array}$ de https://elcomercio.pe/lima/judiciales/elias-cuba-organizacion-criminal-empezocampana-2014-afirma-fiscal-noticia-547287 [Consulta: 15 de mayo del 2019] 
Castilla, O. (2014) El ranking de los secretos. Ojo Público. Recuperado de $\quad$ http://cuentasjuradas.ojo-publico.com/hallazgos/el-ranking-de-los-secretos [Consulta: 15 de mayo del 2019]

Camou, A. (2001). Los desafíos de la gobernabilidad. Estudio preliminar y compilación México: Flacso. Recuperado de https://issuu.com/jooselitoparker/docs/los_desaf_os_de_la_gobernabilidad

Dosek, T. (2015). Presentación. Revista de Ciencia Política Politai, 6(10), 7-8.

Durand, F. (2016) Cuando el poder extractivo captura el Estado. Lobbies, puertas giratorias y paquetazo ambiental en el Perú. Lima: Oxford Committee for Famine Relief.

.Recuperado de: https://peru.oxfam.org/sites/peru.oxfam.org/files/file_attachments/capturadurand \%20VF.pdf

De Soto, H. Ghersi, E., Ghibellini, M., \& Vargas Llosa, M. (1987). El otro sendero : la revolución informal. ILD. Recuperado de https://www.elcato.org/pdf_files/PrologoVargas-Llosa.pdf

El Comercio (2018) Año 2018: los gobernadores y alcaldes detenidos por estar involucrados en delitos. Recuperado de https://elcomercio.pe/peru/ano-2018gobernadores-alcaldes-detenidos-involucrados-delitos-noticia-582732 [Consulta: 15 de mayo del 2019]

Espinoza A. \& Fort, R. (2017) Desarrollo urbano sin planificación territorial: La calidad de la inversión pública en los barrios vulnerables de Lima. Lima: Grupo de Análisis para el Desarrollo. Recuperado de http://www.grade.org.pe/wpcontent/uploads/DICalidadInversionPublica enprensa.pdf

Falleti, T. (2010). Decentralization and Subnational Politics in Latin America. Nueva York, NY: Cambridge University Press.

Helmke, G., \& Levitsky, S. (2006). Informal institutions and democracy: lessons from Latin America [Instituciones informales y democracia: lecciones desde América Latina] Baltimore, M.D.: Johns Hopkins University Press/Universidad de Johns Hopkins 
Huntington, S. P. (2006). Orden politico en sociedades cambiantes [Political order in changing societies]. New Heaven: Yale University Press. Recuperado de http://search.ebscohost.com/login.aspx?direct=true\&db=cat02225a\&AN=pucp.6099 09\&lang=es\&site=eds-live\&scope=site University Press

La República (2019) Se reactiva caso Ecoteva y fiscalía prepara acusación. Recuperado de https://larepublica.pe/politica/1446211-reactiva-caso-ecoteva-fiscalia-preparaacusacion/ [Consulta: 15 de mayo del 2019]

Manrique \& Zegarra, J. (eds.), Cátedra Arequipa. Tercera Sesión. Gestión y Políticas Públicas en Gobiernos Regionales (pp. 123-143). Arequipa: Instituto Gobierno y Desarrollo Humano y Adrus D\&L Editores.

Matos, M. J. (2004) Desborde popular y crisis del Estado. Lima: Fondo Editorial de Congreso del Perú.

Meléndez, C. (2014) Roba pero hace obra. Perú 21. Recuperado de https://peru21.pe/opinion/roba-obra-185915 [Consulta 01 de agosto del 2019]

Miyashiro, J., Wiese, C. \& Marcés, E. (2016) Desigualdad urbana en Lima Metropolitana. En Desigualdad y desarrollo (pp. 333-369). Lima: Centro de Estudios y Promoción de Desarrollo. Recuperado de http://urbano.org.pe/descargas/investigaciones/PERU-HOY/PH_dic16_vf.pdf

Muñoz, P. (2005) El diseño institucional municipal 1980-2004 y sus implicancias para las zonas rurales. Lima: Asociación Servicios Educativos Rurales.

Muñoz, P. (2010). ¿La política importa? Los determinantes políticos de la eficiencia del gasto municipal. Lima: Asociación Servicios Educativos Rurales. Recuperado de https://www.cies.org.pe/sites/default/files/investigaciones/informe_final_paula_m unoz.pdf

Muñoz, P. et al. (2011). El nuevo poder en las regiones. Lima: Pontificia Universidad Católica del Perú. Recuperado de http://blog.pucp.edu.pe/blog/wpcontent/uploads/sites/38/2011/03/peru-debate_final_final.pdf

Muñoz, P. (2014). Gobernabilidad y desarrollo subnacional: problemas de coordinación interinstitucional. Lima: Centro de Investigación Universidad del 
Pacífico. Recuperado de http://agenda2014.pe/publicaciones/agenda2014gobernabilidad-desarrollo-subnacional-2.pdf

Muñoz, P. (2016). El entusiasmo de los que no entusiasman: descentralización y competencia electoral en el Perú. En Perú Hoy, Sin paradero final (pp. 91-109). Lima: DESCO. Recuperado de: http://www.desco.org.pe/sin-paradero-final-serieperu-hoy-n\%C2\%BA-33-julio-2018

Plataforma Anticorrupción (2017) Sospecha generalizada de corrupción contra gobernadores y alcaldes del país. Informe Especial de la PPEDC. Recuperado de https://plataformaanticorrupcion.pe/wp-content/uploads/2017/07/INFORMECORRUPCION-SOBRE-GOBERNADORES-Y-ALCALDES.pdf [Consulta 01 de agosto del 2019]

Procuraduría Pública Especializada en Delitos de Corrupción (PPEDC). Informe de gestión 2017. Recuperado de https://procuraduriaanticorrupcion.minjus.gob.pe/wpcontent/uploads/2018/08/INF ORME-DE-GESTION-2017.pdf [Consulta: 15 de mayo del 2019]

(2018) La corrupción en los gobiernos regionales y locales. Recuperado de https://procuraduriaanticorrupcion.minjus.gob.pe/wpcontent/uploads/2018/09/LA-CORRUPCI\%C3\%93N-EN-GOBIERNOSREGIONALES-Y-LOCALES.pdf [Consulta: 15 de mayo del 2019] Informe de gestión 2018. Recuperado de https://procuraduriaanticorrupcion.minjus.gob.pe/wpcontent/uploads/2019/06/INF ORME-DE-GESTI\%C3\%93N-2018-v04.pdf [Consulta: 15 de mayo del 2019]

Puchuri, F. \& Solis, E. (2019) Convergencias de la corrupción y crimen organizado en el Perú. Recuperado de http://idehpucp.pucp.edu.pe/analisis/convergencias-de-lacorrupcion-y-crimen-organizado-en-el-peru/ [Consulta: 01 de agosto del 2019]

Quiñones, P. (2012). Una mirada al proceso de descentralización tras el primer año de gobierno de Ollanta Humala. Revista Argumentos, (3), 1-10. Recuperado de https://revistaargumentos.iep.org.pe/wpcontent/uploads/2014/04/quiNones_julio2012 .pdf [Consulta: 15 de mayo de 2019] 
Radio Programas del Perú (RPP). (2014) Procuraduría: 92\% de alcaldes del Perú son investigados por corrupción. de https://rpp.pe/politica/actualidad/procuraduria-92-de-alcaldes-del-peru-soninvestigados-por-corrupcion-noticia-708399 [Consulta: 01 de agosto del 2019]

Ramírez, M. (2017) ¿Desde cuándo rige la norma que prohíbe la reelección inmediata de alcalde y gobernadores regionales? Perú 21. Recuperado de https://peru21.pe/politica/rige-norma-prohibe-reeleccion-inmediata-alcaldesgobernadores-regionales-379059 [Consulta: 15 de mayo del 2019]

Remy, M. I. (2010). Elecciones Regionales 2010 o el sueño de la candidatura propia. Revista Argumentos, 3.

Ruiz, M. (2016). Experiencias de gestión en un gobierno regional. En P. Sosa (ed.), Desde adentro: casos prácticos de gestión en el estado peruano (pp. 61-96). Lima, Perú: Instituto de Estudios Peruanos.

Salazar, E. (2016) Felipe Castillo retiró candidatura presidencial pero no puede huir de pedido fiscal de cárcel. Ojo Público. Recuperado de https://ojopublico.com/159/felipe-castillo-retiro-su-candidatura-a-la-presidencia-pero-nopuede-huir-de-pedido-fiscal-de-carcel [Consulta: 15 de mayo del 2019]

Seifert, M. (2014) Colapso de los partidos nacionales y auge de los partidos regionales: las elecciones regionales y municipales 2002-2010. Lima: Pontificia $\begin{array}{llll}\text { Universidad } & \text { Católica } & \text { del } & \text { Perú. }\end{array}$ de http://escuela.pucp.edu.pe/gobierno/wp-content/uploads/2015/04/Colapso-de-lospartidos-nacionales-y-auge-de-los-partidos-regionales-M.-Seifert.pdf

Tanaka, M. (2005). Democracia sin partidos en Perú 2000-2005: Los problemas de representación y las propuestas de reforma política. Lima: Instituto de Estudios Peruanos

Tanaka, M. (2007). El sistema de partidos realmente existente en el Perú, los desafíos de la construcción de una representación política nacional y cómo enrumbar la reforma política. Lima, Perú: Centro de Investigación Económica y Social. Recuperado de https://es.scribd.com/document/40316828/El-sistema-de-partidosrealmente-existente-en-el-Peru-los-desafios-de-la-construccion-de-unarepresentacion-politica-nacional-y-como-enrumbar-la-r 
Tuesta, F., Bensa, J., Campos, M., Muñoz, P. \& Tanaka, M. (2018). Informe Final de la Comisión de Alto Nivel para la Reforma Política. Lima: Presidencia del Consejo de Ministros. Recuperado de https://www.gob.pe/institucion/presidencia/informespublicaciones/267698-informe-final-de-la-comision-de-alto-nivel-para-la-reformapolitica [Consulta: 15 de mayo de 2019]

Vera, S. (2010). Radiografía a la política en las regiones: tendencias a partir de la evidencia de tres procesos electorales. Revista Argumentos, 5. 